\title{
Three-boson recombination at ultralow temperatures
}

\author{
M. T. Yamashita \\ Universidade Estadual Paulista, 18409-010, Itapeva, SP, Brazil \\ T. Frederico \\ Departamento de Física, Instituto Tecnológico de Aeronáutica, 12228-900, São \\ José dos Campos, SP, Brazil \\ Lauro Tomio \\ Instituto de Física Teórica, Universidade Estadual Paulista, 01405-900, São \\ Paulo, Brazil
}

\begin{abstract}
The effects of trimer continuum resonances are considered in the three-body recombination rate of a Bose system at finite energies for large and negative two-body scattering lengths $(a)$. The thermal average of the rate allows to apply our formula to Bose gases at ultra-low temperatures. We found a good quantitative description of the experimental three-body recombination length of cesium atoms to deeply bound molecules up to $500 \mathrm{nK}$. Consistent with the experimental data, the increase of the temperature moves the resonance peak of the three-body recombination rate to lower values of $|a|$ exhibiting a saturation behavior.
\end{abstract}

Key words: Other Bose-Einstein condensation phenomena, three-body recombination, scattering of atoms and molecules, few-body systems PACS: 03.75.Nt, 36.40.-c, 34.50.-s, 21.45.+v

Efimov states [1] result from a general phenomenon that occurs in three-boson systems, corresponding to the appearance of an increasing number of bound states as the two-body binding energy tends to zero. The number is infinite when the two-body energy is zero. So, for systems close to an infinite two-body scattering length some curious three-boson dynamical effects are expected to

Email addresses: yamashita@itapeva.unesp.br (M. T. Yamashita), tobias@ita.br (T. Frederico), tomio@ift. unesp.br (Lauro Tomio). 
appear due to the existence of weakly bound trimers or continuum resonances. Presently the universal properties of three-boson systems can be exploited in its plenitude in ultracold atomic traps by varying from positive to negative values the two-body scattering length $(a)$ near a Feshbach resonance.

Recent experimental observation of a signature of Efimov physics was reported in Ref. [2], considering an ultracold gas of cesium atoms in a trap. Such results definitely open a window to study the universal behavior of three-body systems with large scattering lengths in a controlled way, and can be considered as an experimental verification of correlations among few-body observables with short range interactions with respect to the scattering length.

A giant three-body recombination loss for a large and negative two-body scattering length, where the three-body state hits the continuum threshold [3], was reported in Ref. [2]. For a particular value of the scattering length, characteristic of their experiment, the three-body state attains zero binding energy and produces a huge increase in the measured recombination rate when three free-atoms with low kinetic energy collide forming deeply bound dimers. The increase of the gas temperature $T$ from $10 \mathrm{nK}$ to $250 \mathrm{nK}$ depletes the recombination peak moving it to lower values of $|a|$. This behavior indicates that the zero energy bound state evolves to a triatomic continuum resonance [4], in agreement with results obtained for the trajectory of an Efimov state in the complex plane, i.e., the zero energy trimer dives into the continuum forming a resonance when $|a|$ is increased [4].

Theoretically, the position and width of the triatomic resonance moves to larger values as $|a|$ diminishes, starting from the one that allows a zero energy state [4]. This agrees with the observation that as the temperature raises the peak moves to smaller $|a|$. The average energy available for the recombination process raises with temperature, consequently the recombination peak (roughly at the resonance position) moves toward smaller values of $|a|$ and the intensity diminishes due to the increase of the decay rate to the continuum states. The resonance decay to the three-body continuum states competes with the formation of the deeply bound dimer.

The theory for calculating the zero temperature recombination rate $[3,5]$ represents fairly well the $10 \mathrm{nK}$ data. The issue now is how to describe quantitatively the temperature dependence of the recombination rate and the peak dislocation toward smaller $|a|$ by raising $T$.

In the present work, we generalize the formula of Braaten and Hammer for the recombination rate into deeply bound states. The expression, derived for the particular case of zero kinetic energies $[3,5]$ is extended to positive energies. We include the effect of continuum trimer resonances for $a<0$ in the recombination rate. A thermal average of the recombination rate is performed before 
quantitative comparison with the experimental results of Kraemer et al. [2] for the cesium gas at $T=10,200$ and $250 \mathrm{nK}$. The key point to generalize the rate for positive energies, is to write down the energy and width of continuum resonances in terms of $a<0$ and a three-body scale. To obtain the detailed form of these functions, we solved numerically the renormalized zero-range threebody model $[4,6,7]$ in the complex plane to compute the continuum resonance energy and width as a function of $a$ and a three-body scale.

In practice it is needed one three-body information to set the scale of a real system. For example, in the experiment of Ref. [2] performed with a $10 \mathrm{nK}$ trapped cesium atoms near a Feshbach resonance there is an evidence of the virtual formation of a zero energy state, during the three-atom recombination process to a deeply bound dimer. The recombination peak was found at the particular value of $a$ in their fitting procedure. This information is enough to set the three-body scale.

The three-body physical scale is given by the binding energy $\left(B_{3}\right)$ of the shallowest trimer just below the zero-energy Efimov state. The trimer binding energy $B_{3}$ is calculated from the universal relation [4]

$$
a_{-}^{-1}=0.0297 \sqrt{\frac{m}{\hbar^{2}} B_{3}}
$$

where $\hbar^{2} / m=48.12 / m \mathrm{~K}^{2}$, and $m$ is the boson mass number. In the following general discussion, the three-body scale will be given by the particular value of the scattering length $-a_{-}$, where the three-boson system has an excited Efimov state at zero energy.

The recombination rate, for a finite energy $E$, can be written as

$$
L_{3}(E)=3 C(a, E) \frac{\hbar a^{4}}{m}
$$

where $C(a, E)$ at zero energy is given by [5]:

$$
C(a, 0)=4590 \frac{\sinh \left(2 \eta_{-}\right)}{\sin ^{2}\left[s_{0} \ln \left(|a| / a_{-}\right)\right]+\sinh ^{2} \eta_{-}},
$$

where $\eta_{-}$is a dimensionless quantity that characterizes the resonance width. It describes the unknown decay rate of Efimov states into deeply bound dimer states plus a free atom.

Equations (2), with $L_{3} \equiv L_{3}(0)$, and (3) were used in Ref. [2] to fit the 
recombination length $\rho_{3}$ at zero energy, as

$$
\rho_{3}=\left[\frac{2 m}{\sqrt{3} \hbar} L_{3}\right]^{\frac{1}{4}}
$$

The finite energy extension of Eq. (3) is done by the inclusion of the pole brought by the continuum resonance.

With the trimer at zero energy, the S-matrix pole is obtained from

$$
\zeta\left(a, a_{-}\right)=\sin \left[s_{0} \ln \left(|a| / a_{-}\right)\right]
$$

when $a=-a_{-}\left(\zeta\left(a, a_{-}\right)=0\right)$. Here and in the following, $a_{-} \equiv a_{-}(E=0)$ to simplify the notation. In a naive extension of the above function to the complex energy plane, the corresponding zeros, $\zeta\left(a, a_{-}(E)\right)=0$, will give the energy and partial width of the continuum resonances related to the scattering length $a=-a_{-}(E)$.

In extending Eq. (3) to non-zero energies, it is assumed that the parameter $\eta_{-}$, which characterizes the width of the resonance, varies slowly with the scattering energy. This implies that it is not expected an appreciable effect when the trimer moves from the threshold to an ultralow energy continuum resonance. However, the calculated decay width of the resonances to continuum states has a magnitude large enough to be important for the observed results.

The slow variation of $\eta_{-}$in terms of the energy is expected because it corresponds to the formation of a deeply bound dimer with a size much smaller than $|a|$. Therefore, the short-range part of the triatomic wave function should be responsible for the recombination process, which is deeply inside the potential, such that does not strongly depend on the wave function tail, determined by the large scattering length.

The problem now is focused on how to get $a_{-}$as a function of energy. Or, how to obtain $a_{-}(E)$. The resonance energy and partial width scale with $a$ and $a_{-}$ ( $a_{-}$gives the dependence on the three-body scale). Therefore, we can write that

$$
E=\frac{\hbar^{2}}{m a_{-}^{2}} \mathcal{F}\left(a / a_{-}\right)
$$

where the scaling function $\mathcal{F}\left(a / a_{-}\right)$should be universal in the limit of large values of $a$ and $a_{-}$, with respect to the interaction range. Note that the imaginary part of $E$ in Eq.(6) gives the decay width to the continuum states. For $a=-a_{-}$the trimer has zero energy implying that $\mathcal{F}(-1)=0$. By expanding 
the real and imaginary parts of $\mathcal{F}(z)$ around $z=-1$, we obtain the approximate formula

$$
\frac{m a_{-}^{2}}{\hbar^{2}} E \equiv \epsilon=\alpha\left(1+\frac{a}{a_{-}}\right)-\mathrm{i} \gamma\left(1+\frac{a}{a_{-}}\right)^{2}
$$

where $\alpha$ and $\gamma$ are fitting parameters. This expression is found to reach reasonable results when comparing to the numerical results of the model presented in Ref. [4].

In figure 1 we show the dimensionless products $\epsilon_{R}=\operatorname{Re}(\epsilon)$ and $\epsilon_{I}=\operatorname{Im}(\epsilon)$ as functions of $a / a_{-}$, for the three-body energy scale derived from the condition of an Efimov state at zero energy for $a=-a_{-}$. The simple parameterization is compared to the calculated values using a renormalized zero-range three-boson model [4], with the three-body scale adjusted to allow an Efimov state at zero energy. The real part of the resonance energy behaves almost linearly for the range of values of $a / a_{-}$shown in the figure, while the imaginary part of $\epsilon$ has an almost quadratic dependence on $a / a_{-}$. The values of the dimensionless constants of Eq. (7), $\alpha=1.01$ and $\gamma=3.58$, are chosen to fit the numerical results obtained with the renormalized zero range model of Ref. [4].

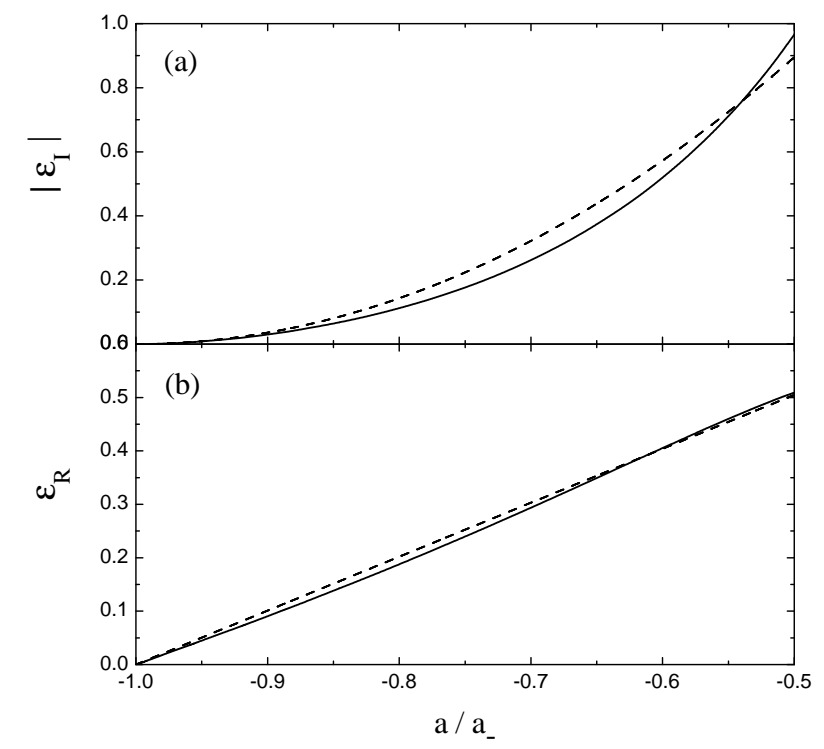

Fig. 1. Real, $\epsilon_{R}$ (lower frame (b)), and imaginary, $\epsilon_{I}$ (upper frame (a)), parts of the dimensionless expression for $\epsilon \equiv E\left(m a_{-}^{2} / \hbar^{2}\right)$ as a function of $\left(a / a_{-}\right)$. The solid lines are obtained from the renormalized zero model [4] and the dashed lines are the corresponding analytic results given by Eq. (7) with $\alpha=1.01$ and $\gamma=3.58$.

One can easily find the function $a_{-}(E)$ by solving Eq.(7) for a given value of $E$, with $a=-a_{-}(E)$ : 


$$
a_{-}(E)=a_{-}\left[1+\mathrm{i} \frac{\alpha}{2 \gamma}\left(1-\sqrt{1-4 \mathrm{i} \frac{\gamma}{\alpha^{2}} \frac{m a_{-}^{2}}{\hbar^{2}} E}\right)\right] .
$$

Equation (8) is strictly valid for $E$ being the resonance energy with the imaginary part related to the partial width to the continuum states, in spite of that we perform a naive analytic continuation of Eq. (8) to values of $E$ along the real axis. Then, instead of (5), we will have

$$
\zeta\left(a, a_{-}(E)\right)=\sin \left[s_{0} \ln \left(\frac{|a|}{a_{-}+\Delta(E)}\right)\right],
$$

with

$$
\Delta(E)=\mathrm{i} a_{-} \frac{\alpha}{2 \gamma}\left(1-\sqrt{1-4 \mathrm{i} \frac{\gamma}{\alpha^{2}} \frac{m a_{-}^{2}}{\hbar^{2}} E}\right) .
$$

The function $\zeta\left(a, a_{-}(E)\right)$ has a zero at the resonance energy for $a=-a_{-}(E)$ when the decay width to the deep states vanishes. This extends the Efimov law of appearance of bound states to the case of continuum resonances as a function of the scattering length. Scaling $a$ by $\exp \left(\pi / s_{0}\right)$, and considering that $\alpha$ and $\gamma$ are scale invariants, the zero of $\zeta\left(a_{-}(E), a\right)$ gives a resonance energy re-scaled by a factor of $\exp \left(-2 \pi / s_{0}\right)$.

The recombination rate for finite energy is derived from the original expression at $E=0$ by introduction of the complex function for $\zeta\left(a_{-}(E), a\right)$, given by Eq. (9), assuming that the main effect for the three-body recombination into deep dimer states comes from the change of the nearest S-matrix pole due to the triatomic continuum resonances, while the $\eta_{-}$is a slowly varying function of $E$. The resulting analytic expression for $L_{3}(E)$ in Eq. (2) is given by

$$
C(a, E)=4590 \frac{\sinh \left(2 \eta_{-}\right)}{\left|\zeta\left(a_{-}(E), a\right)+i \sinh \eta_{-}\right|^{2}}
$$

The next step is to perform a thermal average of the recombination rate $\left\langle L_{3}\right\rangle_{T}$. Considering that the bosons are not condensed, we will use a classical thermal energy distribution, which allows the separation of the center of mass motion. The remaining degrees of freedom in the center of mass kinetic energy $E$ are the Jacobi energies of the two independent relative motions, then $E=E_{p}+E_{q}$, where the index $p$ and $q$ are related, respectively, to the relative momentum of a pair of atoms, $\vec{p}$, and the relative momentum of the third atom to the pair, $\vec{q}$. The thermal average of the recombination rate calculated classically, with $\beta \equiv 1 /(k T)$ and $k$ the Boltzmann constant, is given by 


$$
\left\langle L_{3}\right\rangle_{T}=\frac{4}{\pi} \beta^{3} \int_{0}^{\infty} d E_{p} \sqrt{E_{p}} \int_{0}^{\infty} d E_{q} \sqrt{E_{q}} L_{3}\left(E_{p}+E_{q}\right) e^{-\left[\beta\left(E_{p}+E_{q}\right)\right]}
$$

The three-body observables are correlated to the two- and three-body reference scales in the Thomas-Efimov limit, where the ratio $a / r_{0}\left(r_{0}\right.$ is the effective range) goes to infinity (scaling limit). These correlations are generally defined in terms of functions of dimensionless quantities named scaling functions. Using $a_{-}$as a reference, an observable can be written in terms of a scaling function [7], such that

$$
\mathcal{O}(E)=\left(a_{-}\right)^{\nu} \mathcal{F}\left(\epsilon, a / a_{-}\right)
$$

where $\mathcal{O}$ is a general observable of the three-body system at energy $E$ with the power $\nu$ giving the proper dimension to the observable. The function $\mathcal{F}\left(\epsilon, a / a_{-}\right)$converges to a limiting curve when the Thomas-Efimov condition is approached [7]. The scaling functions, calculated in the the scaling limit [8], are identified with a limit cycle [9].

From the above Eq. (13), the thermal average of the scaling function of a given observable is

$$
\langle\mathcal{O}(E)\rangle_{T}=a_{-}^{\nu}\left\langle\mathcal{F}\left(\epsilon, a / a_{-}\right)\right\rangle_{T} \equiv\left(a_{-}\right)^{\nu} \mathcal{F}_{t h}\left(\frac{m a_{-}^{2}}{\hbar^{2}} k T, a / a_{-}\right),
$$

which defines a correlation between $a$ and $T$ for a given value of $\langle\mathcal{O}\rangle_{T}$ with the reference scale $a_{-}$. The observed peak position of the recombination rate as a function of $T$ is one of such example.

The parameters of the simple curves for the resonance energy enters through Eq. (11) in the calculation of the thermal average of the recombination length,

$$
\left\langle\rho_{3}\right\rangle_{T}=\left[\frac{2 m}{\sqrt{3} \hbar}\left\langle L_{3}\right\rangle_{T}\right]^{\frac{1}{4}}
$$

In figure 2, the results of the present model are compared to the experimental data given in [2]. We allowed a change in the $\eta_{-}$parameter, from 0.06 found in [2] to 0.03 , as we have also considered $a_{-}$to be $0.898 a_{0}$ instead of $0.85 a_{0}$. Replacing $a_{-}$and the cesium mass number $m=133$ in Eq. (1) we found $B_{3}=1.81 \mathrm{mK}$ for the experiment. The experimental data has temperatures around $10 \mathrm{nK}$ for one set (full circles), $200 \mathrm{nK}$ (full triangles) and $250 \mathrm{nK}$ (open diamonds). Our result for $200 \mathrm{nK}$ is somewhat above the data, while for $300 \mathrm{nK}$ approaches the experimental values. The increase of temperature makes the resonance peak lower and wider, almost disappearing for $500 \mathrm{nK}$. 


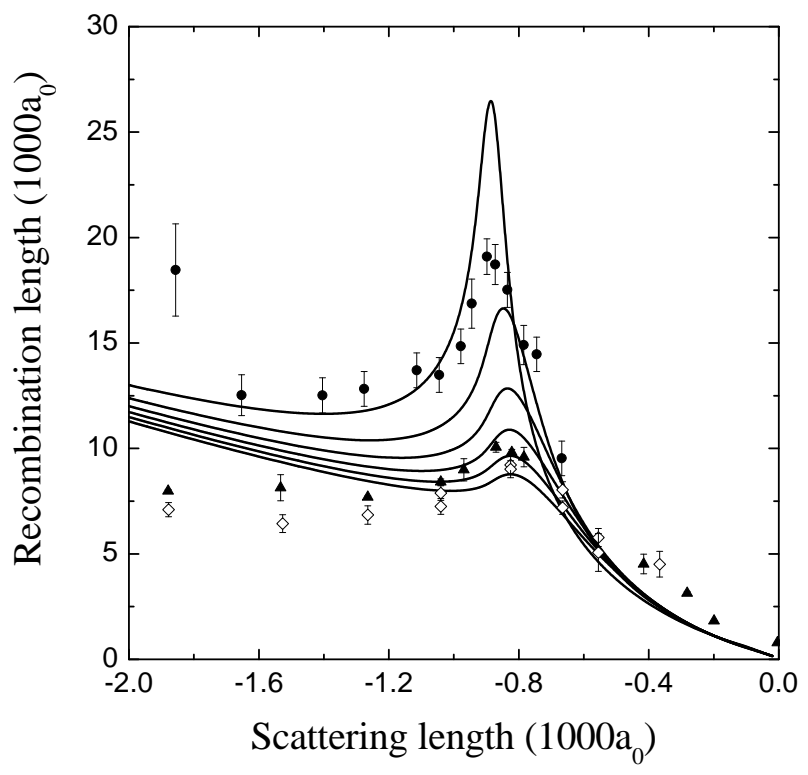

Fig. 2. Recombination length $\left\langle\rho_{3}\right\rangle_{T}$ [as given by Eq. (15)] in a trapped gas of cesium atoms, as a function of the scattering length and temperature. The solid curves from up to bottom are the theoretical results for $T=10 \mathrm{nK}, 100 \mathrm{nK}, 200 \mathrm{nK}, 300 \mathrm{nK}$, $400 \mathrm{nK}$ and $500 \mathrm{nK}$. The symbols are the experimental results for $T=10 \mathrm{nK}$ (full circles), $200 \mathrm{nK}$ (full triangles) and $250 \mathrm{nK}$ (open diamonds) from [2].

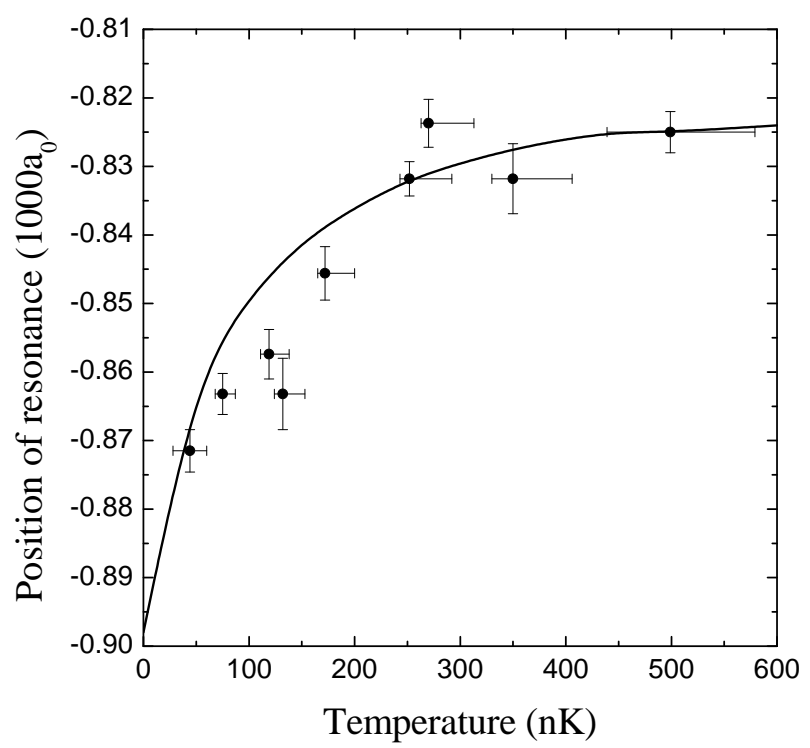

Fig. 3. Position of the maximum of the recombination length as a function of the temperature. For the experimental data, see comments in [10].

The movement of the peak to lower values of $|a|$ is also seen in the figure. The present simplified model of introducing temperature dependence in the threebody recombination rate is also compatible with results obtained in Ref. [11].

In figure 3, we show the results for the scattering length corresponding to the peak position as a function of temperature compared to recent and preliminary 
experimental results [10]. In the present model, no new parameters are used to obtain these results.

In summary, for a three-boson system, it was derived an extension to positive energies of an expression given in [3] for the recombination length, including the triatomic continuum resonance pole. The position and width of the triatomic resonance increase as the modulus of the scattering length is decreased near the critical condition for which an Efimov state turns into a continuum resonance.

The thermal average of the observables defines new scaling functions appropriate to ultracold gases. Our approach was tested with experimental data of Ref. [2], as shown in Figs. 2 and 3. We found that for the particular thermal average of the recombination rate into deep dimer states, the gross features of the experimental data for $T$ below $500 \mathrm{nK}$ are reproduced, with parameters set at $T=0$. The scattering length for the peak position as a function of temperature describes nicely the data and the saturation for $T$ above $200 \mathrm{nK}$ is well described.

To conclude, the trajectory of the triatomic continuum resonance is reflected in the correlation curve of $a$ and $T$, which is a direct consequence of dominance of just two scales: the two and three body ones which determine the three-body observables. Therefore, the thermal scaling law was checked by the experiment with cesium atoms which strongly supports the dominance of few scales in the physics of ultracold atoms. Such universal thermal scaling law for three-body recombination length into deep dimers are expected to be confirmed also in other systems.

In view of the nice results obtained in comparison with cesium experimental data, this simplified analytical approach of introducing temperature dependence in few-body thermal observables for ultracold atomic gases is shown to give the basic properties of a more detailed formalism to be developed.

We thank Dr. R. Grimm and T. Kraemer for useful discussion and for providing us preliminary data referred in [10]. This work was partially supported by Fundação de Amparo a Pesquisa do Estado de São Paulo and Conselho Nacional de Desenvolvimento Científico e Tecnológico.

\section{References}

[1] V. Efimov, Phys. Lett. B 33, 563 (1970); Nucl. Phys. A362, 45 (1981); V. Efimov, Comm. Nucl. Part. Phys. 19, 271 (1990) and references therein.

[2] T. Kraemer et al, Nature 440, 315 (2006). 
[3] E. Braaten and H.-W. Hammer, Phys. Rev. Lett. 87, 160407 (2001).

[4] F. Bringas, M.T. Yamashita and T. Frederico. Phys. Rev. A 69, 040702(R) (2004).

[5] E. Braaten, H.-W. Hammer, Phys. Rep. 428, 259 (2006).

[6] S.K. Adhikari, T. Frederico, and I.D. Goldman, Phys. Rev. Lett. 74 (1995) 487; S.K. Adhikari and T.Frederico, Phys. Rev. Lett. 74, 4572 (1995).

[7] M.T. Yamashita, T. Frederico, A. Delfino, and L. Tomio, Phys. Rev. A 66, 052702 (2002).

[8] T. Frederico, L. Tomio, A. Delfino, and A. E. A. Amorim, Phys. Rev. A 60, R9 (1999).

[9] R. F. Mohr, R. J. Furnstahl, H. -W. Hammer, R. J. Perry and K. G. Wilson, Ann. Phys. 321, 225 (2006).

[10] Preliminary experimental data were presented at the 18th International IUPAP Conference on Few-Body Problems in Physics, in Santos, Brazil, August 26, 2006. Such data were also provided to the authors by R. Grimm and T. Kraemer in private communications.

[11] S. Jonsell, Europhys. Lett. 76, 8 (2006). 